\title{
EFFECT OF SILICA FUME ON THE STRENGTH OF CEMENT MORTAR
}

\author{
Harjinder Singh $^{1}$, Shikha Bansal ${ }^{2}$ \\ ${ }^{1}$ ME Highways, PEC University of technology, Chandigarh, India \\ ${ }^{2}$ ME Highways, PEC University of technology, Chandigarh, India
}

\begin{abstract}
The replacement of sand/cement by certain percentage of silica fumes, resulted in the improvement in compressive strength of the mortar. Silica fumes to the highly pozzolanic materials because it consists essentially of silica in non-crystalline form with a high specific surface. It is used to improve the mechanical properties of the concrete. The main objective of this paper is to study the effect of silica fume on the compressive strength of mortar. Three proportions of mixes viz mix 1:3, mix 1:4 and mix 1:6 with different percentages of silica fumes replacement with sand/cement were used. The maximum increase in strength at the age of 28 days when sand is replaced by $15 \%$ of silica fume has been observed as $40 \%$ and in case of cement replaced with $15 \%$ of silica fume, the observed increase in compressive strength of mortar comes out to be $28 \%$.
\end{abstract}

A quantitative cost analysis shows that with the replacement of cement and sand by silica fume, the in cost is more when sand is replaced and it is less when cement is replaced.

Keywords: pozzolanic, silica fumes, non- crystalline, compressive strength

\section{INTRODUCTION}

With the increase in cement/mortar, now a days in infrastructure projects, high cost of such projects put higher emphasis on huge durability. Normal concrete/mortar is week in tension, has limited ductility and little resistance to cracking. The micro cracks propogates with the application of load which lead to brittle failure of concrete. The research and investigation has been carried out to develop a material which can better perform in comparison to conventional concrete.

\subsection{High Performance Concrete}

High performance concrete is one such type of concrete which has excellent performance in terms of strength, ease of placement and compaction without segregation enhanced durability in adverse climatic conditions, enhancement of long term mechanical properties, toughness which can be obtained with the incorporation of minerals admixtures like fly ash, silica fume, rice husk ash etc. Addition of silica fume improves the durability of concrete and improvement in its ability to protect embedded steel from corrosion.

\subsection{Silica Fume}

The term "microsillica" is adopted to characterize the silica fumes which are used for production of concrete/ mortar. Microsilica or condensed silica fume (CSF) is a byproduct resulting from reduction of high purity quartz with coal in the electric arc furnace used in the manufacture of silicone, ferrosilicon and other alloys of silicone, CSF which is highly pozzolanic has high content of amorphous silicon dioxide in the range of $85 \%$ to $98 \%$ in the form of very fine spherical particles averaging diameters about 0.1 to 0.5 microns. The microsilica is about 100 times finer than ordinary cement, there are serious problems associated with its transportation, storage, handeling and dispersing in the concrete.

\subsubsection{Physical and Chemical Properties of Silica} Fume

Silica fume is very fine off white pozzolanic material processed from naturally occurring amorphous silica deposit in New Zealand's Rotoura district. Uniformity of product quality is ensured by careful blending of raw materials and stringent process control. The general physical and chemical properties of silica fume are given in Table 1.1 and Table 1.2

Table1.1-Physical Properties of Silica Fume

\begin{tabular}{|l|l|}
\hline Colour & Varies from light to dark grey \\
\hline Specific gravity & 2.1 to 2.3 \\
\hline Bulk loose density & 230 to $300 \mathrm{~kg} / \mathrm{m}^{3}$ \\
\hline Fineness & $20,000 \mathrm{~m}^{2} / \mathrm{kg}$ \\
\hline Partical size & $0.1 \mu \mathrm{m}$ (approx) \\
\hline
\end{tabular}

Table1.2-Chemical Properties of Silica Fume

\begin{tabular}{|l|l|}
\hline Constituent & \%age \\
\hline $\mathrm{SiO}_{2}$ & $90-96$ \\
\hline $\mathrm{Al}_{2} \mathrm{O}_{3}$ & $0.5-0.8$ \\
\hline $\mathrm{Fe}_{2} \mathrm{O}_{3}$ & $0.2-0.8$ \\
\hline $\mathrm{MgO}$ & $0.5-1.5$ \\
\hline $\mathrm{CaO}$ & $0.1-0.5$ \\
\hline $\mathrm{Na}_{2} \mathrm{O}$ & $0.2-0.7$ \\
\hline $\mathrm{K}_{2} \mathrm{O}$ & $0.4-1.0$ \\
\hline
\end{tabular}




\section{NEED AND OBJECTIVE}

\subsection{Need}

From all the studies which has been carried out about the silica fume as a partial replacement of cement, sufficient work shows the long term and the short term strength development of silica fume concrete/mortar. However, little work has been carried out on the strength characteristics on silica fume modified mortar when silica fume is replaced by weight of part of sand/cement. Thus it was considered necessary to investigate the effect of silica fume on strength of cementitious material when part of cement/sand is replaced by certain percentage of silica fume.

\subsection{Objective}

The present work has been undertaken to study the following aspects of silica fume modified mortar.

1. The effects of silica fume on compressive strength of mortar at the age of 3, 7 and 28 days when a part of cement is replaced by silica fume.

2. The effects of silica fume on compressive strength of mortar at the age of 3, 7 and 28 days when a part of sand is replaced by silica fume.

\section{LITRATURE}

Although the use of use of silica fume in concrete has increased significantly in past few years, its beneficial properties were not well realized until compressive research was undertaken in late 70 's and early 80 's to study the influence of silica fumes on concrete properties.

One of the first major structures to incorporate silica fume was in Sweden where silica fume concrete was used in tower construction of the new Tijron cable - stayed bridge that was completed in late 1981. Part of the cement was replaced by silica fume to decrease the thermal stresses in massive section without reducing strength.

Sellevoid et al (1987) studied that during the hydration for the sealed cement paste, there is decrease in relative humidity and chemical shrinkage. The Portland cement paste incorporated $0.8 \%$ and $1.6 \%$ condensed silica fume and had water- cement plus silica fume ratio ranging between $0.2,0.3$ and 0.4 . they founded that relative humidity (RH) decreased rapidly during the first two weeks and reached about $78 \% \mathrm{RH}$ after more than a year for the lowest $\mathrm{W} /(\mathrm{C}+\mathrm{SF})$ paste, the value for the highest $\mathrm{W} /(\mathrm{C}+\mathrm{SF})$ being about $87 \%$ RH.

In 1982, the sale of silica fume in Qurbec, Canada started picking up because of the easily availability of this product. One of the major uses of silica fume concrete in US was the rehabilitation of the stilling basin of Kinzua dam in 1983 . The concrete contain $386 \mathrm{~kg} / \mathrm{m}^{3}$ of cement with $70 \mathrm{~kg} / \mathrm{m}^{3}$ of silica fume and the specified compressive strength was 70 $\mathrm{MPa}$ at 7 days and $86 \mathrm{MPa}$ at 28 days. After three years compressive strength of concrete was reported to be 110 $\mathrm{MPa}$. Since this large scale project silica fume concrete has progressively gained acceptance in wide range of application of USA.

The other application of silica fume in cement based material world wide are bank vault construction, parking garage repairs, overlays for bridge decks, spillways, stilling basins and water canals, under water repairs, light weight concrete construction, high strength concrete in high used structures, cements grouts for filling post tensioning ducts, high pressure concrete pipes and more recently off- shore platform construction because of the improved chemical resistance of the silica fume concrete. It has also been used in the construction aluminum and magnesium plants, water and waste water treatment facilities as well as paper mill plants.

According to Haque M.N(1996), the incorporation of silica fume in concrete decrease the drying shrinkage and Cabrera et al (1990) shows that the permeability decreases with the addition of silica fume to concrete. Punkki et al (1996) reported that the loss of workability due to the use of silica fume creates the difficulty to utilize silica fume concrete accurately.

\section{EXPERIMENTATION AND TESTING}

In order to study the effect of silica fume on compressive strength of cement mortar, the silica fume is to be added in different percentages (i.e. $5 \%, 10 \%, 15 \%$ by weight) replacing the equivalent quantity of cement and sand separately. The different proportion of mortar 1:3, 1:4 and 1:6 has been selected for the study. For each mix the compressive strength of $(7.06 \times 7.06 \times 7.06)$ cube is to be recorded for different percentages of silica fume (cement/sand replaced) for particular mix, average compressive strength of three similar cubes has to be noted.

\subsection{Mix Proportion}

\subsubsection{Mix 1 (Sand Replaced by Silica Fume)}

Control mix

Cement $(\mathrm{C})=1 \mathrm{~kg}$

Water $(\mathrm{W})=0.5 \mathrm{KG}$

$\mathrm{SF}(\mathrm{P}) \quad=0$

Aggregate/sand $(\mathrm{A})=3 \mathrm{~kg}$

\section{Water to cement ratio $(\mathrm{W} / \mathrm{C})=\mathbf{0 . 5 0}$}

Silica fume to cement ratio $(\mathrm{P} / \mathrm{C})=0.00$

When SF replaced sand three content of SF was selected

1. Cement $+5 \% \mathrm{SF}+95 \%$ sand $\left(\mathrm{SFA}_{1}\right)$

2. Cement $+10 \% \mathrm{SF}+90 \%$ sand $\left(\mathrm{SFA}_{2}\right)$

3. Cement $+15 \% \mathrm{SF}+85 \%$ sand $\left(\mathrm{SFA}_{3}\right)$

\subsubsection{Mix 2(Cement Replaced by Silica Fume)}

Similarly for cement replacement the same content were selected.

1. $95 \%$ cement + sand $+5 \% \mathrm{SF}\left(\mathrm{SFC}_{1}\right)$

2. $90 \%$ cement + sand $+10 \% \mathrm{SF}\left(\mathrm{SFC}_{2}\right)$

3. $85 \%$ cement + sand $+15 \% \mathrm{SF}\left(\mathrm{SFC}_{3}\right)$ 
The same proportion were repeated for the other types of mixes like 1:4 and 1:6 in the same pattern as detailed above and for each type of mix cubes mould were cast and their compressive strength were noted.

\section{RESULTS}

Table 5.1 Compressive Strength of mortar for Control Mix WATER CEMENT RATIO: 0.5

\begin{tabular}{|c|c|c|c|c|c|c|c|c|c|}
\hline \multirow[t]{2}{*}{ CEMENT : SAND } & \multicolumn{3}{|c|}{3 DAYS } & \multicolumn{3}{|c|}{7 DAYS } & \multicolumn{3}{|c|}{28 DAYS } \\
\hline & $\begin{array}{l}\text { Load } \\
\text { in } \\
\text { tonne }\end{array}$ & $\begin{array}{l}\text { Comp. } \\
\text { st. in } \\
\mathrm{N} / \mathbf{m m}^{2}\end{array}$ & $\begin{array}{l}\text { Avg. } \\
\text { Comp. } \\
\text { st. in } \\
\mathrm{N} / \mathrm{mm}^{2}\end{array}$ & $\begin{array}{l}\text { Load } \\
\text { in } \\
\text { tonne }\end{array}$ & $\begin{array}{l}\text { Comp. } \\
\mathrm{st} \text { in } \\
\mathrm{N} / \mathrm{mm}^{2}\end{array}$ & $\begin{array}{l}\text { Avg. } \\
\text { Comp. } \\
\text { st. in } \\
\mathrm{N} / \mathrm{mm}^{2}\end{array}$ & $\begin{array}{l}\text { Load } \\
\text { in } \\
\text { tonne }\end{array}$ & $\begin{array}{l}\text { Comp. } \\
\text { st. in } \\
\mathrm{N} / \mathbf{m m}^{2}\end{array}$ & $\begin{array}{l}\text { Avg. } \\
\text { Comp. } \\
\text { st. in } \\
\mathrm{N} / \mathrm{mm}^{2}\end{array}$ \\
\hline \multirow[t]{3}{*}{$1: 3$} & 12.80 & 25.60 & \multirow{3}{*}{25.86} & 17.50 & 35.00 & \multirow{3}{*}{35.00} & 24.75 & 49.50 & \multirow{3}{*}{50.16} \\
\hline & 13.50 & 27.00 & & 17.00 & 34.00 & & 25.50 & 51.00 & \\
\hline & 12.50 & 25.00 & & 18.00 & 36.00 & & 25.0 & 50.00 & \\
\hline \multirow[t]{3}{*}{ 1:4 } & 9.00 & 18.00 & \multirow{3}{*}{18.66} & 12.50 & 25.00 & \multirow{3}{*}{24.20} & 17.00 & 34.00 & \multirow{3}{*}{34.33} \\
\hline & 9.75 & 19.50 & & 11.80 & 23.60 & & 17.50 & 35.00 & \\
\hline & 9.25 & 18.50 & & 12.00 & 24.00 & & 17.00 & 34.00 & \\
\hline \multirow[t]{3}{*}{$1: 6$} & 6.00 & 12.00 & \multirow{3}{*}{12.83} & 8.50 & 17.00 & \multirow{3}{*}{17.00} & 12.80 & 25.60 & \multirow{3}{*}{26.2} \\
\hline & 6.75 & 13.50 & & 8.00 & 16.00 & & 13.50 & 27.00 & \\
\hline & 6.50 & 13.00 & & 9.00 & 18.00 & & 13.00 & 26.00 & \\
\hline
\end{tabular}

Table 5.2 Compressive Strength of Mortar (Sand replaced with Silica Fume)

MIX 1:3(CEMENT: SAND)

WATER CEMENT RATIO: 0.5

\begin{tabular}{|c|c|c|c|c|c|c|c|c|c|}
\hline & \multicolumn{3}{|c|}{3 DAYS } & \multicolumn{3}{|c|}{7 DAYS } & \multicolumn{3}{|c|}{28 DAYS } \\
\hline & $\begin{array}{l}\text { Load } \\
\text { in } \\
\text { tonne }\end{array}$ & $\begin{array}{l}\text { Comp. } \\
\text { st. in } \\
\mathrm{N} / \mathrm{mm}^{2}\end{array}$ & $\begin{array}{l}\text { Avg. } \\
\text { Comp. } \\
\text { st. in } \\
\mathrm{N} / \mathrm{mm}^{2}\end{array}$ & $\begin{array}{l}\text { Load } \\
\text { in } \\
\text { tonne }\end{array}$ & $\begin{array}{l}\text { Comp. } \\
\text { st. in } \\
\mathrm{N} / \mathrm{mm}^{2}\end{array}$ & $\begin{array}{l}\text { Avg. } \\
\text { Comp. } \\
\text { st. in } \\
\mathrm{N} / \mathrm{mm}^{2}\end{array}$ & $\begin{array}{l}\text { Load } \\
\text { in } \\
\text { tonne }\end{array}$ & $\begin{array}{l}\text { Comp. } \\
\text { st. in } \\
\mathrm{N} / \mathrm{mm}^{2}\end{array}$ & $\begin{array}{l}\text { Avg. } \\
\text { Comp. } \\
\text { st. in } \\
\mathrm{N} / \mathrm{mm}^{2}\end{array}$ \\
\hline \multirow{3}{*}{$\begin{array}{l}\text { Cement + 5\% SF + 95\% } \\
\text { Sand }\end{array}$} & 17.00 & 34.00 & \multirow{3}{*}{33} & 19.50 & 39.00 & \multirow{3}{*}{39} & 31.00 & 62.00 & \multirow{3}{*}{61.67} \\
\hline & 16.50 & 33.00 & & 19.00 & 38.00 & & 30.50 & 61.00 & \\
\hline & 16.00 & 32.00 & & 20.00 & 40.00 & & 31.00 & 62.00 & \\
\hline \multirow{3}{*}{$\begin{array}{l}\text { Cement }+10 \% \text { SF }+ \\
90 \% \text { Sand }\end{array}$} & 17.50 & 35.00 & \multirow{3}{*}{34.36} & 23.00 & 46.00 & \multirow{3}{*}{47.20} & 33.75 & 67.50 & \multirow{3}{*}{68.83} \\
\hline & 18.00 & 36.00 & & 24.00 & 48.00 & & 35.20 & 70.00 & \\
\hline & 16.50 & 33.00 & & 23.80 & 47.60 & & 34.50 & 69.00 & \\
\hline \multirow{3}{*}{$\begin{array}{l}\text { Cement }+15 \% \text { SF + } \\
\text { 85\% Sand }\end{array}$} & 17.80 & 35.60 & \multirow{3}{*}{36.03} & 24.75 & 49.50 & \multirow{3}{*}{49.02} & 34.80 & 69.60 & \multirow{3}{*}{70.20} \\
\hline & 18.75 & 37.50 & & 23.80 & 47.60 & & 35.50 & 71.00 & \\
\hline & 17.50 & 35.00 & & 25.00 & 50.00 & & 35.00 & 70.00 & \\
\hline
\end{tabular}

Table 5.3 Compressive Strength of Mortar (Cement replaced with Silica Fume)

MIX 1:3(CEMENT: SAND)

WATER CEMENT RATIO: 0.5

\begin{tabular}{|c|c|c|c|c|c|c|c|c|c|}
\hline & \multicolumn{3}{|c|}{3 DAYS } & \multicolumn{3}{|c|}{7 DAYS } & \multicolumn{3}{|c|}{28 DAYS } \\
\hline & $\begin{array}{l}\text { Load } \\
\text { in } \\
\text { tonne }\end{array}$ & $\begin{array}{l}\text { Comp. } \\
\text { st. in } \\
\mathrm{N} / \mathrm{mm}^{2}\end{array}$ & $\begin{array}{l}\text { Avg. } \\
\text { Comp. } \\
\text { st. in } \\
\mathrm{N} / \mathrm{mm}^{2}\end{array}$ & $\begin{array}{l}\text { Load } \\
\text { in } \\
\text { tonne }\end{array}$ & $\begin{array}{l}\text { Comp. } \\
\text { st. in } \\
\mathrm{N} / \mathrm{mm}^{2}\end{array}$ & $\begin{array}{l}\text { Avg. } \\
\text { Comp. } \\
\text { st. in } \\
\mathrm{N} / \mathrm{mm}^{2}\end{array}$ & $\begin{array}{l}\text { Load } \\
\text { in } \\
\text { tonne }\end{array}$ & $\begin{array}{l}\text { Comp. } \\
\text { st. in } \\
\mathrm{N} / \mathbf{m m}^{2}\end{array}$ & $\begin{array}{l}\text { Avg. } \\
\text { Comp. } \\
\text { st. in } \\
\mathrm{N} / \mathrm{mm}^{2}\end{array}$ \\
\hline \multirow{3}{*}{$\begin{array}{l}95 \% \text { Cement }+5 \% \text { SF + } \\
\text { Sand }\end{array}$} & 15.00 & 30.00 & \multirow{3}{*}{30.86} & 18.75 & 37.50 & \multirow{3}{*}{36.34} & 29.50 & 59.00 & \multirow{3}{*}{59.00} \\
\hline & 15.50 & 31.00 & & 18.00 & 36.00 & & 29.00 & 58.00 & \\
\hline & 15.80 & 31.60 & & 17.75 & 35.50 & & 30.00 & 60.00 & \\
\hline $90 \%$ Cement $+10 \%$ SF & 18.75 & 37.50 & & 18.75 & 37.50 & & 33.75 & 67.50 & \\
\hline
\end{tabular}




\begin{tabular}{|c|c|c|c|c|c|c|c|c|c|}
\hline \multirow[t]{2}{*}{ + Sand } & 18.00 & 36.00 & \multirow[t]{2}{*}{36.34} & 18.00 & 36.00 & \multirow[t]{2}{*}{36.34} & 35.20 & 70.00 & \multirow[t]{2}{*}{68.83} \\
\hline & 17.75 & 35.50 & & 17.75 & 35.50 & & 34.50 & 69.00 & \\
\hline \multirow{3}{*}{$\begin{array}{l}85 \% \text { Cement }+15 \% \text { SF } \\
+ \text { Sand }\end{array}$} & 14.00 & 28.00 & \multirow{3}{*}{27.66} & 21.00 & 42.00 & \multirow{3}{*}{40.33} & 32.00 & 64.00 & \multirow{3}{*}{64.36} \\
\hline & 13.50 & 27.00 & & 20.00 & 40.00 & & 32.80 & 65.60 & \\
\hline & 14.00 & 28.00 & & 19.50 & 39.00 & & 31.75 & 63.50 & \\
\hline
\end{tabular}

Table 5.4 Compressive Strength of Mortar (Sand replaced with Silica Fume) MIX 1:4 (CEMENT: SAND)

WATER CEMENT RATIO: 0.5

\begin{tabular}{|c|c|c|c|c|c|c|c|c|c|}
\hline & \multicolumn{3}{|c|}{3 DAYS } & \multicolumn{3}{|c|}{7 DAYS } & \multicolumn{3}{|c|}{28 DAYS } \\
\hline & $\begin{array}{l}\text { Load } \\
\text { in } \\
\text { tonne }\end{array}$ & $\begin{array}{l}\text { Comp. } \\
\text { st. in } \\
\mathrm{N} / \mathrm{mm}^{2}\end{array}$ & $\begin{array}{l}\text { Avg. } \\
\text { Comp. } \\
\text { st. in } \\
\mathrm{N} / \mathrm{mm}^{2}\end{array}$ & $\begin{array}{l}\text { Load } \\
\text { in } \\
\text { tonne }\end{array}$ & $\begin{array}{l}\text { Comp. } \\
\text { st. in } \\
\mathrm{N} / \mathrm{mm}^{2}\end{array}$ & $\begin{array}{l}\text { Avg. } \\
\text { Comp. } \\
\text { st. in } \\
\mathrm{N} / \mathrm{mm}^{2}\end{array}$ & $\begin{array}{l}\text { Load } \\
\text { in } \\
\text { tonne }\end{array}$ & $\begin{array}{l}\text { Comp. } \\
\text { st. in } \\
\mathrm{N} / \mathrm{mm}^{2}\end{array}$ & $\begin{array}{l}\text { Avg. } \\
\text { Comp. } \\
\text { st. in } \\
\mathrm{N} / \mathrm{mm}^{2}\end{array}$ \\
\hline \multirow{3}{*}{$\begin{array}{l}\text { Cement + 5\% SF + 95\% } \\
\text { Sand }\end{array}$} & 11.50 & 23.00 & \multirow{3}{*}{23.86} & 14.75 & 29.50 & \multirow{3}{*}{29.16} & 21.00 & 42.00 & \multirow{3}{*}{42.16} \\
\hline & 12.50 & 25.00 & & 15.00 & 30.00 & & 21.50 & 43.00 & \\
\hline & 11.80 & 23.60 & & 14.00 & 28.00 & & 20.75 & 41.50 & \\
\hline \multirow{3}{*}{$\begin{array}{l}\text { Cement }+10 \% \text { SF + } \\
90 \% \text { Sand }\end{array}$} & 12.00 & 24.00 & \multirow{3}{*}{24.86} & 16.50 & 33.00 & \multirow{3}{*}{32.17} & 22.75 & 45.50 & \multirow{3}{*}{45.83} \\
\hline & 12.50 & 25.00 & & 15.75 & 31.00 & & 23.50 & 47.00 & \\
\hline & 12.80 & 25.60 & & 16.00 & 32.00 & & 22.50 & 45.00 & \\
\hline \multirow{3}{*}{$\begin{array}{l}\text { Cement + 15\% SF + } \\
\text { 85\% Sand }\end{array}$} & 12.50 & 25.00 & \multirow{3}{*}{26.00} & 17.00 & 34.00 & \multirow{3}{*}{34.20} & 23.00 & 46.00 & \multirow{3}{*}{47.00} \\
\hline & 13.50 & 27.00 & & 16.80 & 33.60 & & 24.00 & 48.00 & \\
\hline & 13.00 & 26.00 & & 17.50 & 35.00 & & 23.50 & 47.00 & \\
\hline
\end{tabular}

Table 5.5 Compressive Strength of Mortar- (Cement replaced with Silica Fume)

MIX 1:4(CEMENT: SAND)

WATER CEMENT RATIO: 0.5

\begin{tabular}{|c|c|c|c|c|c|c|c|c|c|}
\hline & \multicolumn{3}{|c|}{3 DAYS } & \multicolumn{3}{|c|}{7 DAYS } & \multicolumn{3}{|c|}{28 DAYS } \\
\hline & $\begin{array}{l}\text { Load } \\
\text { in } \\
\text { tonne }\end{array}$ & $\begin{array}{l}\text { Comp. } \\
\text { st. in } \\
\mathrm{N} / \mathrm{mm}^{2}\end{array}$ & $\begin{array}{l}\text { Avg. } \\
\text { Comp. } \\
\text { st. in } \\
\mathrm{N} / \mathrm{mm}^{2}\end{array}$ & $\begin{array}{l}\text { Load } \\
\text { in } \\
\text { tonne }\end{array}$ & $\begin{array}{l}\text { Comp. } \\
\text { st. in } \\
\mathrm{N} / \mathbf{m m}^{2}\end{array}$ & $\begin{array}{l}\text { Avg. } \\
\text { Comp. } \\
\text { st. in } \\
\mathrm{N} / \mathrm{mm}^{2}\end{array}$ & $\begin{array}{l}\text { Load } \\
\text { in } \\
\text { tonne }\end{array}$ & $\begin{array}{l}\text { Comp. } \\
\text { st. in } \\
\mathrm{N} / \mathbf{m m}^{2}\end{array}$ & $\begin{array}{l}\text { Avg. } \\
\text { Comp. } \\
\text { st. in } \\
\mathrm{N} / \mathrm{mm}^{2}\end{array}$ \\
\hline \multirow{3}{*}{$\begin{array}{l}\text { 95\% Cement }+5 \% \text { SF + } \\
\text { Sand }\end{array}$} & 10.00 & 20.00 & \multirow{3}{*}{21.67} & 12.80 & 25.60 & \multirow{3}{*}{24.87} & 20.00 & 40.00 & \multirow{3}{*}{40.50} \\
\hline & 11.50 & 23.00 & & 12.00 & 24.00 & & 20.75 & 41.50 & \\
\hline & 11.00 & 22.60 & & 12.50 & 25.00 & & 20.00 & 40.00 & \\
\hline \multirow{3}{*}{$\begin{array}{l}\text { 90\% Cement + } 10 \% \text { SF } \\
+ \text { Sand }\end{array}$} & 10.50 & 21.00 & \multirow{3}{*}{26.00} & 13.50 & 27.00 & \multirow{3}{*}{34.20} & 20.50 & 41.00 & \multirow{3}{*}{47.00} \\
\hline & 10.80 & 21.60 & & 12.75 & 25.50 & & 21.00 & 42.00 & \\
\hline & 10.00 & 21.00 & & 13.00 & 26.00 & & 20.80 & 41.60 & \\
\hline \multirow{3}{*}{$\begin{array}{l}85 \% \text { Cement + 15\% SF } \\
+ \text { Sand }\end{array}$} & 09.50 & 19.00 & \multirow{3}{*}{20.00} & 14.00 & 28.00 & \multirow{3}{*}{27.00} & 21.50 & 43.00 & \multirow{3}{*}{43.34} \\
\hline & 10.50 & 21.00 & & 13.50 & 27.00 & & 22.00 & 44.00 & \\
\hline & 10.00 & 20.00 & & 13.00 & 26.00 & & 21.50 & 43.00 & \\
\hline
\end{tabular}

Table 5.6 Compressive strength of Mortar (Sand replaced with Silica Fume)

MIX 1:6(CEMENT: SAND)

WATER CEMENT RATIO: 0.5

\begin{tabular}{|c|c|c|c|c|c|c|c|c|c|}
\hline & \multicolumn{3}{|c|}{3 DAYS } & \multicolumn{3}{|c|}{7 DAYS } & \multicolumn{3}{|c|}{28 DAYS } \\
\hline & $\begin{array}{l}\text { Load } \\
\text { in } \\
\text { tonne }\end{array}$ & $\begin{array}{l}\text { Comp. } \\
\text { st. in } \\
\mathrm{N} / \mathrm{mm}^{2}\end{array}$ & $\begin{array}{l}\text { Avg. } \\
\text { Comp. } \\
\text { st. in } \\
\mathrm{N} / \mathrm{mm}^{2}\end{array}$ & $\begin{array}{l}\text { Load } \\
\text { in } \\
\text { tonne }\end{array}$ & $\begin{array}{l}\text { Comp. } \\
\text { st. in } \\
\mathrm{N} / \mathbf{m m}^{2}\end{array}$ & $\begin{array}{l}\text { Avg. } \\
\text { Comp. } \\
\text { st. in } \\
\mathrm{N} / \mathrm{mm}^{2}\end{array}$ & $\begin{array}{l}\text { Load } \\
\text { in } \\
\text { tonne }\end{array}$ & $\begin{array}{l}\text { Comp. } \\
\text { st. in } \\
\mathrm{N} / \mathrm{mm}^{2}\end{array}$ & $\begin{array}{l}\text { Avg. } \\
\text { Comp. } \\
\text { st. in } \\
\mathrm{N} / \mathrm{mm}^{2}\end{array}$ \\
\hline \multirow{3}{*}{$\begin{array}{l}\text { Cement + 5\% SF + 95\% } \\
\text { Sand }\end{array}$} & 7.50 & 15.00 & \multirow{3}{*}{16.00} & 10.50 & 21.00 & \multirow{3}{*}{20.86} & 15.50 & 31.00 & \multirow{3}{*}{31.34} \\
\hline & 8.50 & 17.00 & & 09.80 & 19.60 & & 16.50 & 33.00 & \\
\hline & 8.80 & 16.00 & & 11.00 & 22.00 & & 15.00 & 30.00 & \\
\hline \multirow{2}{*}{$\begin{array}{l}\text { Cement }+10 \% \text { SF + } \\
\text { 90\% Sand }\end{array}$} & 7.80 & 15.60 & \multirow[b]{2}{*}{16.37} & 11.00 & 22.00 & \multirow[b]{2}{*}{22.00} & 16.50 & 33.00 & \multirow[b]{2}{*}{45.83} \\
\hline & 8.75 & 17.50 & & 10.50 & 21.00 & & 17.50 & 35.00 & \\
\hline
\end{tabular}




\begin{tabular}{|c|c|c|c|c|c|c|c|c|c|}
\hline & 8.00 & 25.60 & & 11.50 & 23.00 & & 16.80 & 33.60 & \\
\hline \multirow{3}{*}{$\begin{array}{l}\text { Cement }+15 \% \text { SF + } \\
\text { 85\% Sand }\end{array}$} & 8.00 & 16.00 & \multirow{3}{*}{16.86} & 11.50 & 23.00 & \multirow{3}{*}{22.84} & 17.50 & 35.00 & \multirow{3}{*}{35.34} \\
\hline & 8.80 & 17.60 & & 10.75 & 21.50 & & 18.00 & 36.00 & \\
\hline & 8.50 & 17.00 & & 12.00 & 24.00 & & 17.50 & 35.00 & \\
\hline
\end{tabular}

Table 5.7 Compressive strength of Mortar- (Cement replaced with Silica Fume)

MIX 1:6(CEMENT: SAND)

WATER CEMENT RATIO: 0.5

\begin{tabular}{|c|c|c|c|c|c|c|c|c|c|}
\hline & \multicolumn{3}{|c|}{3 DAYS } & \multicolumn{3}{|c|}{7 DAYS } & \multicolumn{3}{|c|}{28 DAYS } \\
\hline & $\begin{array}{l}\text { Load } \\
\text { in } \\
\text { tonne }\end{array}$ & $\begin{array}{l}\text { Comp. } \\
\text { st. in } \\
\mathrm{N} / \mathrm{mm}^{2}\end{array}$ & $\begin{array}{l}\text { Avg. } \\
\text { Comp. } \\
\text { st. in } \\
\mathrm{N} / \mathrm{mm}^{2}\end{array}$ & $\begin{array}{l}\text { Load } \\
\text { in } \\
\text { tonne }\end{array}$ & $\begin{array}{l}\text { Comp. } \\
\text { st. in } \\
\mathrm{N} / \mathbf{m m}^{2}\end{array}$ & $\begin{array}{l}\text { Avg. } \\
\text { Comp. } \\
\text { st. in } \\
\mathrm{N} / \mathbf{m m}^{2}\end{array}$ & $\begin{array}{l}\text { Load } \\
\text { in } \\
\text { tonne }\end{array}$ & $\begin{array}{l}\text { Comp. } \\
\text { st. in } \\
\mathrm{N} / \mathrm{mm}^{2}\end{array}$ & $\begin{array}{l}\text { Avg. } \\
\text { Comp. } \\
\text { st. in } \\
\mathrm{N} / \mathrm{mm}^{2}\end{array}$ \\
\hline \multirow{3}{*}{$\begin{array}{l}\text { 95\% Cement + 5\% SF + } \\
\text { Sand }\end{array}$} & 07.00 & 14.00 & \multirow{3}{*}{14.84} & 08.75 & 17.50 & \multirow{3}{*}{17.66} & 14.75 & 29.50 & \multirow{3}{*}{30.16} \\
\hline & 07.50 & 15.00 & & 08.50 & 17.00 & & 15.50 & 31.00 & \\
\hline & 07.75 & 15.50 & & 09.25 & 18.50 & & 15.00 & 30.00 & \\
\hline \multirow{3}{*}{$\begin{array}{l}90 \% \text { Cement }+10 \% \text { SF } \\
\text { + Sand }\end{array}$} & 06.50 & 13.00 & \multirow{3}{*}{13.84} & 08.80 & 17.60 & \multirow{3}{*}{18.20} & 16.00 & 32.00 & \multirow{3}{*}{31.67} \\
\hline & 07.25 & 14.50 & & 09.00 & 18.00 & & 15.50 & 31.00 & \\
\hline & 07.00 & 14.00 & & 09.50 & 19.00 & & 16.00 & 32.00 & \\
\hline \multirow{3}{*}{$\begin{array}{l}85 \% \text { Cement }+15 \% \text { SF } \\
+ \text { Sand }\end{array}$} & 06.25 & 12.50 & \multirow{3}{*}{13.04} & 09.50 & 19.00 & \multirow{3}{*}{18.86} & 15.75 & 31.50 & \multirow{3}{*}{32.17} \\
\hline & 06.80 & 13.60 & & 08.80 & 17.60 & & 16.50 & 33.00 & \\
\hline & 10.00 & 13.00 & & 10.00 & 20.00 & & 16.00 & 32.00 & \\
\hline
\end{tabular}

\section{CONCLUSION}

On the basis of study carries out above the following conclusion may be drawn-

1. The compressive strength of mortar improves when a part of cement/ sand is replaced by certain percentage of silica fume.

2. The compressive strength of cement/mortar increases in the range varying between $30-40 \%$ with the addition of silica fume by $5-15 \%$ when sand is replaced.

3. The compressive strength of cement/mortar increases in the range varying between $20-30 \%$ with the addition of silica fume by $5-15 \%$ when cement is replaced.

4. The compressive strength of silica fume modified mortar increases by $10 \%$ (approximately) in the case of sand replaced in comparison to cement replacement by equal proportion in the same mix.

5. The increase in compressive strength is found to be maximum for the mix proportion 1:3 for all the percentages of silica fume addition. Therefore, richer mix will be more responsive in addition of silica fume.

6. Modified mortar with silica fume and 53 grade of cement exhibits a maximum increase in compressive strength at the ages of 3 days and 28 days and improvement is less than $15 \%$ at the age of 7 days.

7. The maximum increase in strength is observed at a silica fume content of $15 \%$ when sand is replaced by silica fume.

8. Keeping in view of cost cement replacement will be economical than sand replacement but compressive strength of mortar obtained will be lesser.

\section{REFERENCES}

[1] Haque M.N., "Strength Development and Drying Shrinkage of High-Strength Concretes", Cement \& Concrete Composites Journal, Vol-18,No-5,pp. 333342,1996.

[2] Cabrera J.G.,Claisse P.A., "Measurement of Chloride Penetration into Silica Fume Concrete", Cement and Concrete Composites, 12(3), (1990),pp. 157-161

[3] Punkki J., Golaszewski J., Gjørv O.E., “ Workability Loss of High-Strength Concrete", ACI Materias Journal,Vol. 93,Issue 5, (1996),pp.427-431.

[4] E. Sellevold, Condensed silica fume in concrete, FIP/NB symposium on high strength concrete, Stavanger 1987.

[5] IS-12269-1982 "Specification for 53 grade of cement" BIS, New Delhi.

[6] IS-383-1970 Specifications for coarse and fine aggregates from natural source for concrete BIS, New Delhi.

[7] P.K. Mehta, proceedings of the $3^{\text {rd }}$ international conference on the use of fly ash, silica fume, slag and natural pozzolana in concrete, ACISP- 114, Trondhoim, 1989

[8] Hindy EE, Micro B Omar, C and Aitchin P.S, "Drying shrinkage of ready mixed high performance concrete" ACI Materials Journal vol.91 No. 3 May June 1994 pp 300-305.

[9] Mehta P.K. 1989, Pozzolanic and cementitious byproducts in concrete another look, proceedings $3^{\text {rd }}$ CANMET/ACI international conference on the use of fly ash silica fume, slag and other mineral byproduct in concrete 